\title{
Target fishing of glycopentalone using integrated inverse docking and reverse pharmacophore mapping approach
}

\author{
A.B. Gurung1, M.A. Ali², A. Bhattacharjee ${ }^{1}$, K.M. Al-Anazi ${ }^{3}$, M.A.

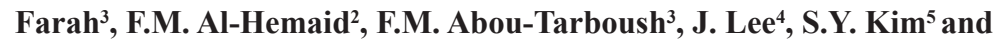 \\ F.S.M. Al-Anazi ${ }^{2}$
}

${ }^{1}$ Department of Biotechnology and Bioinformatics, North Eastern Hill University, Shillong, Meghalaya, India

${ }^{2}$ Department of Botany and Microbiology, College of Science, King Saud University, Riyadh, Saudi Arabia

${ }^{3}$ Department of Zoology, College of Science, King Saud University, Riyadh, Saudi Arabia

${ }^{4}$ Department of Environment and Forest Resources,

Chungnam National University, Daehak-ro, Yuseong-gu, Daejeon,

Republic of South Korea

${ }^{5}$ International Biological Material Research Center,

Korea Research Institute of Bioscience and Biotechnology, Daejeon,

Republic of South Korea

Corresponding authors: M.A. Ali / A. Bhattacharjee

E-mail: alimohammad@ksu.edu.sa / atanubioinfo@gmail.com

Genet. Mol. Res. 15 (3): gmr.15038544

Received February 11, 2016

Accepted April 8, 2016

Published August 12, 2016

DOI http://dx.doi.org/10.4238/gmr.15038544

Copyright (C) 2016 The Authors. This is an open-access article distributed under the terms of the Creative Commons Attribution ShareAlike (CC BY-SA) 4.0 License.

ABSTRACT. Glycopentalone isolated from Glycosmis pentaphylla (family Rutaceae) has cytotoxic and apoptosis inducing effects in various human cancer cell lines; however, its mode of action is not 
known. Therefore, target fishing of glycopentalone using a combined approach of inverse docking and reverse pharmacophore mapping approach was used to identify potential targets of glycopentalone, and gain insight into its binding modes against the selected molecular targets, viz., CDK-2, CDK-6, Topoisomerase I, Bcl-2, VEGFR-2, Telomere:Gquadruplex and Topoisomerase II. These targets were chosen based on their key roles in the progression of cancer via regulation of cell cycle and DNA replication. Molecular docking analysis revealed that glycopentalone displayed binding energies ranging from -6.38 to -8.35 $\mathrm{kcal} / \mathrm{mol}$ and inhibition constants ranging from 0.758 to $20.90 \mu \mathrm{M}$. Further, the binding affinities of glycopentalone to the targets were in the order: Telomere:G-quadruplex $>$ VEGFR-2 $>$ CDK- $6>$ CDK-2 $>$ Topoisomerase II $>$ Topoisomerase I $>$ Bcl-2. Binding mode analysis revealed critical hydrogen bonds as well as hydrophobic interactions with the targets. The targets were validated by reverse pharmacophore mapping of glycopentalone against a set of 2241 known human target proteins which revealed CDK-2 and VEGFR-2 as the most favorable targets. The glycopentalone was well mapped to CDK-2 and VEGFR-2 which involve six pharmacophore features (two hydrophobic centers and four hydrogen bond acceptors) and nine pharmacophore features (five hydrophobic, two hydrogen bond acceptors and two hydrogen bond donors), respectively. The present computational approach may aid in rational identification of targets for small molecules against large set of candidate macromolecules before bioassays validation.

Key words: Glycosmis pentaphylla; Glycopentalone; Molecular docking; Reverse pharmacophore mapping; Anticancer; Cell cycle

\section{INTRODUCTION}

The spread of abnormal cells and uncontrolled cell division, i.e., cancer, is a serious health issue worldwide (Greenlee et al., 2000; Gan et al., 2003; Siegel et al., 2015). Chemotherapy, the use of cytotoxic compounds in the treatment of cancer and radiotherapy have traditionally been used to kill cancer cells, but both these methods in use have also led to serious side effects including anemia, asthenia, leukopenia, low immunity, nausea, and neutropenia (DeSantis et al., 2014). Therefore, exploring natural products for effective drug lead for cancer is of great interest worldwide today. The discovery of a drug is a costly endeavor where a drug under experimentation has to be successfully passed through in-depth studies followed by clinical trials before it can be used as a treatment. In silico approaches such as molecular docking provide rapid ways to assess likely binding compounds with target macromolecules, and is therefore being widely practiced in pharmacology (Stark and Powers, 2012). Inverse docking has proved to be an important computational tool in the identification of novel macromolecular targets for a drug or ligand pertaining to its mechanism of action and/ or side effects (Grinter et al., 2011; Chen and Ren, 2014), and involves the docking of a small ligand or drug in potential binding sites of a set of clinically relevant macromolecular targets (Kharkar et al., 2014). The second approach of target fishing is the reverse pharmacophore

Genetics and Molecular Research 15 (3): gmr.15038544 
mapping approach, which is based on the fitting and mapping of small query molecules against predetermined pharmacophore features of targets (Liu et al., 2010).

Glycosmis pentaphylla (Retz.) Correa (family Rutaceae), commonly known as 'orange berry' or 'gin berry', is used in the treatment of various ailments like anaemia, arthritis, cough, facial inflammation, jaundice and rheumatism (Mohammed et al., 2010). G. pentaphylla has also previously been reported to have variety of biological activities such as antimicrobial (Abbas et al., 2011; Amran et al., 2011); antioxidant (Amran et al., 2011; Gupta et al., 2011); cytotoxic (Amran et al., 2011); hepatoprotective (Nayak et al., 2011); apoptotic (Sreejith et al., 2012; Yang et al., 2014); antiarthritic (Sivakumar et al., 2014); and anti-inflammatory activity (Prawej et al., 2015). Recently, glycopentalone reported from G. pentaphylla has in vitro hepatocellular anticancer activity (Sreejith and Asha, 2015); however, the mode of action is unknown. Hence, in the present study, the molecular docking of glycopentalone with cyclin-dependent protein kinase, DNA topoisomerases, Bcl-2, VEGFR-2 and Telomere:Gquadruplex was performed in order to investigate binding interactions of glycopentalone with key enzymes and receptor proteins associated with cell division and DNA replication.

\section{MATERIAL AND METHODS}

The chemical structure of glycopentalone (Figure 1) was modeled using the software Chemsketch (http://www.acdlabs.com/resources/freeware/), and optimized by a MMFF94 force field using the optimization parameters (500 steepest descent algorithms, convergence criterion 10e-7; Halgren, 1996). The optimized compound was used to perform molecular docking. The three dimensional structures of a total number of seven molecular targets (receptors) viz., CDK-2 (PDB ID:1DI8), CDK-6 (PDB ID:1XO2), Topoisomerase II (PDB ID:1ZXM), Topoisomerase I (PDB ID:1T8I), Bcl-2 (PDB ID:2O2F), VEGFR-2 (PDB ID:2OH4) and Telomere:G-quadruplex (PDB ID: 1L1H), were obtained from Protein Data Bank (PDB) (www.rcsb.org), and prepared for docking following a previously described method (Gurung et al., 2016).

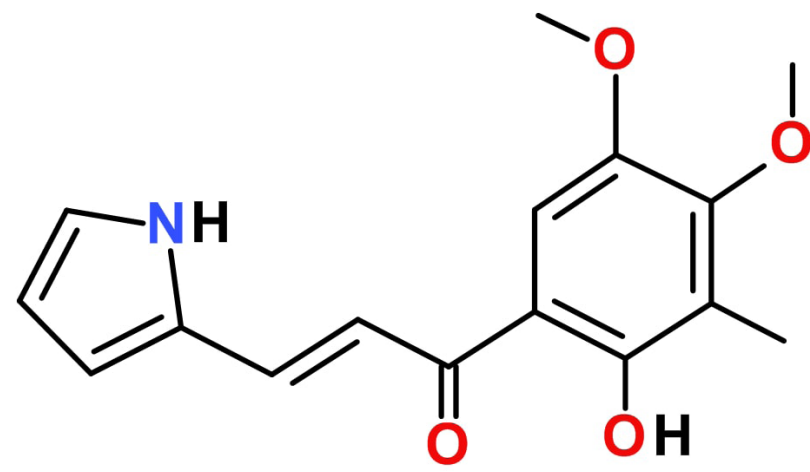

Figure 1. Chemical structure of glycopentalone selected for molecular docking.

The inverse docking of glycopentalone was executed (parameters-initial population of randomly placed individuals: 150 , maximum number of energy evaluations: $2,500,000$, crossover rate: 0.8 , mutation rate: 0.02 , algorithm: lamarckian genetic, independent docking runs: 50) against receptors using AutoDock4.2 (Morris et al., 2009). The conformations that 
differ by $<2.0 \AA$ RMSD (root mean square deviation) were clustered together. The most favorable conformation was represented by the lowest inhibition constant (Ki) and the lowest free energy of binding $(\Delta \mathrm{G})$. The conformation with the lowest $\Delta \mathrm{G}$ was evaluated for molecular interaction with their receptors using LigPlot + v 1.4.5 (Laskowski and Swindells, 2011). The parameters for hydrogen-bond calculations included a maximum hydrogen-acceptor distance of $2.70 \AA$ and maximum donor-acceptor distance of $3.35 \AA$, while the non-bonded contact parameters included a minimum contact distance of $2.90 \AA$ and a maximum contact distance of $3.90 \AA$. To ensure that the binding pose of the docked compound represents a favorable and valid potential binding mode, the docking parameters and methods were validated by redocking the cocrystal ligand in order to see the ability of the AutoDock program to reproduce the orientation and position of the ligand observed in the crystal structure. The figures for docking validation and binding site pockets were captured using UCSF chimera (Pettersen et al., 2004).

\section{Reverse pharmacophore database screening}

The PharmMapper server (http://59.78.96.61/pharmmapper/) was used to validate the targets of glycopentalone based on the pharmacophore mapping approach (Liu et al., 2010). It correctly identified some of the experimentally determined targets of Tamoxifen, used as an adjuvant therapy in the treatment of breast cancer (Hughes-Davies et al., 2009), indicating the reliability of the server for target fishing. Pharmmapper contains 7302 pharmacophore models (2241 annotated as human target proteins) generated using the LigandScout software which extract 3D pharmacophore features such as hydrophobic centre, positive charged centre, negative charged centre, hydrogen bond acceptor, hydrogen bond donor and aromatic ring. There are two steps involved in the reverse pharmacophore mapping methodology employed in the Pharmmapper server: a) flexible alignment of queried small molecules against a pharmacophore model of each target; and b) scoring of aligned poses by calculating fit values between the molecule and the pharmacophore models. We used default parameters of the PharmMapper server, such as: a) conformation generation of query molecule based on the MOEA-based conformation generation algorithm Cyndi (Liu et al., 2009); b) target set chosen as human targets; and c) genetic algorithm was used to optimize pharmacophore mapped poses. The pharmacophore mapped features of glycopentalone were visualized using Discovery Studio 4.1 Visualizer.

\section{RESULTS AND DISCUSSION}

\section{Inverse docking analysis}

The redocking of cocrystal ligands to their respective molecular targets exhibited RMSD of $<2 \AA$ between the original cocrystal ligand position and docked poses as shown in Figure 2. This confirmed that the ligands were bound to their targets very close to the true conformation, indicating the reliability of the docking protocols and parameters. Glycopentalone was docked against seven molecular targets, viz., CDK-2, CDK-6, Topoisomerase I, Bcl-2, VEGFR-2, Telomere:G-quadruplex and Topoisomerase II, to gain insight into their possible binding modes. The active site residue(s), grid box dimensions, binding energies, and inhibition constants of glycopentalone against the selected targets are shown in Tables 1 and 2. The

Genetics and Molecular Research 15 (3): gmr.15038544 
lowest binding poses of the best docked ligands were selected for molecular interactionshydrogen bonds and hydrophobic interactions analysis. The molecular interaction results of glycopentalone are depicted in Figure 3.

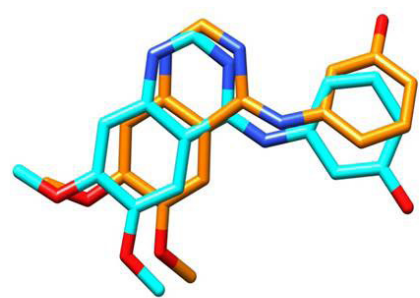

A. RMSD:1.510A[PDB ID:1DI8]

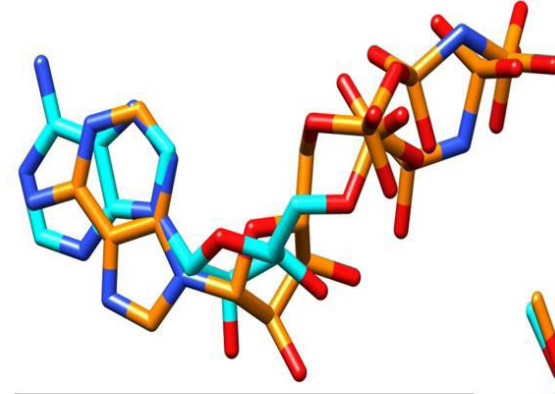

C. RMSD:1.508A[PDB ID:1ZXM]

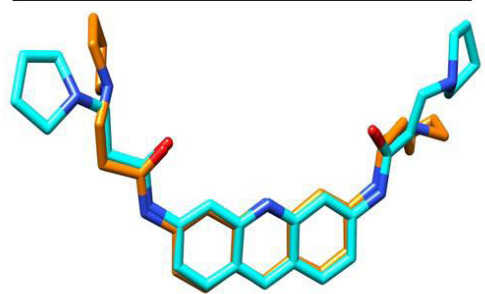

E. RMSD:1.528A[PD ID:1L1H]

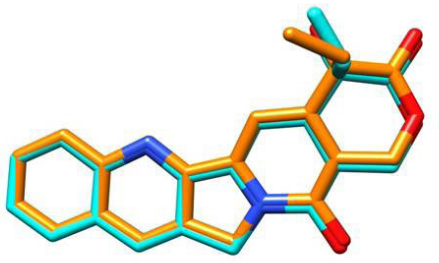

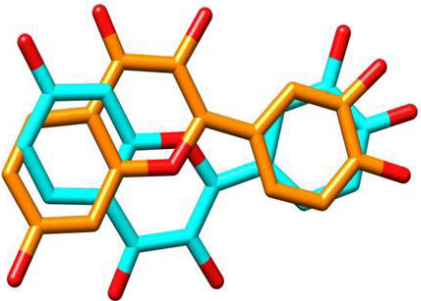

B. RMSD:1.466A[PDB ID:1X02]

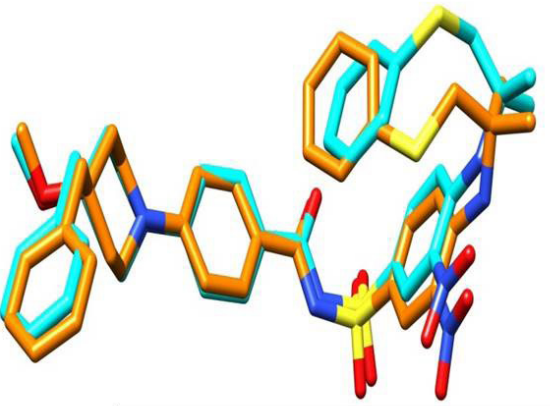

D. RMSD:0.833A[PDB ID:202F]

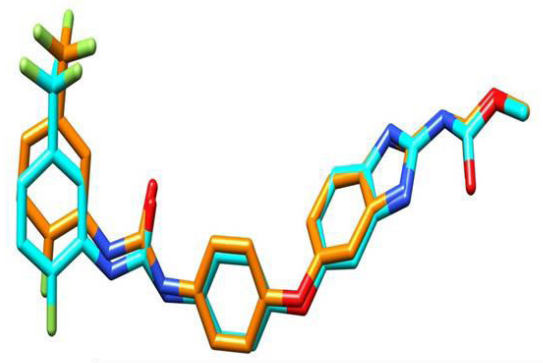

F. RMSD:0.608A[PDB ID:20H4]

\section{G. RMSD:0.255A[PDB ID:1T8I]}

Figure 2. A.-G. Docking validation by redocking cocrystal ligands to their corresponding molecular targets indicated by their PDB IDs. Original conformations of cocrystal ligands are displayed in cyan stick while docked poses are represented in orange stick. Root mean square deviation (RMSD) was calculated between the original and docked poses of cocrystal ligands.

Genetics and Molecular Research 15 (3): gmr.15038544 
A.B. gurung et al.

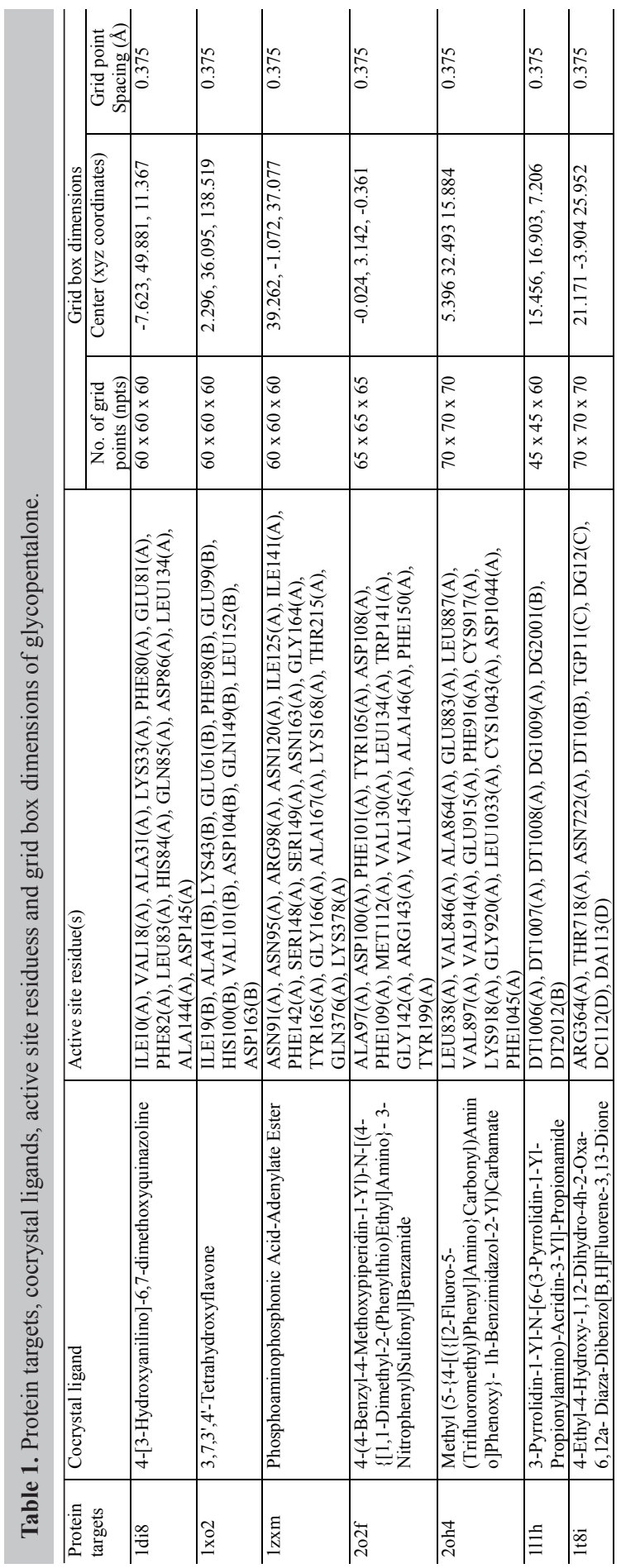

Genetics and Molecular Research 15 (3): gmr.15038544 


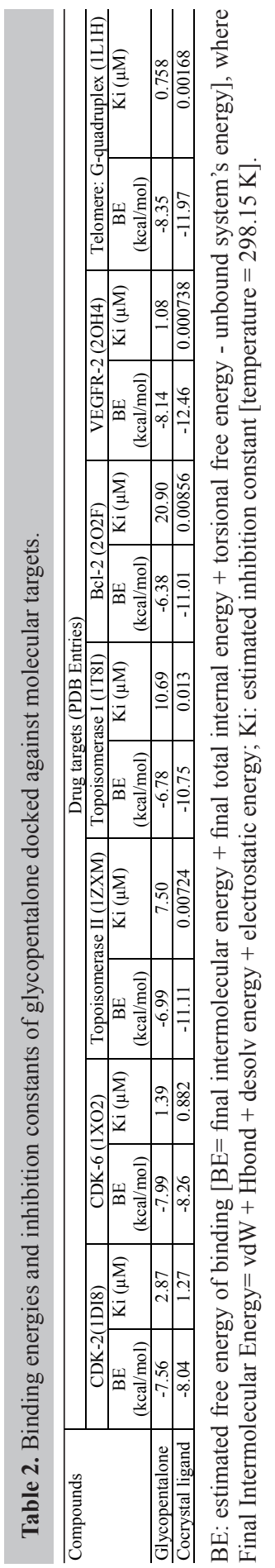

Genetics and Molecular Research 15 (3): gmr.15038544 


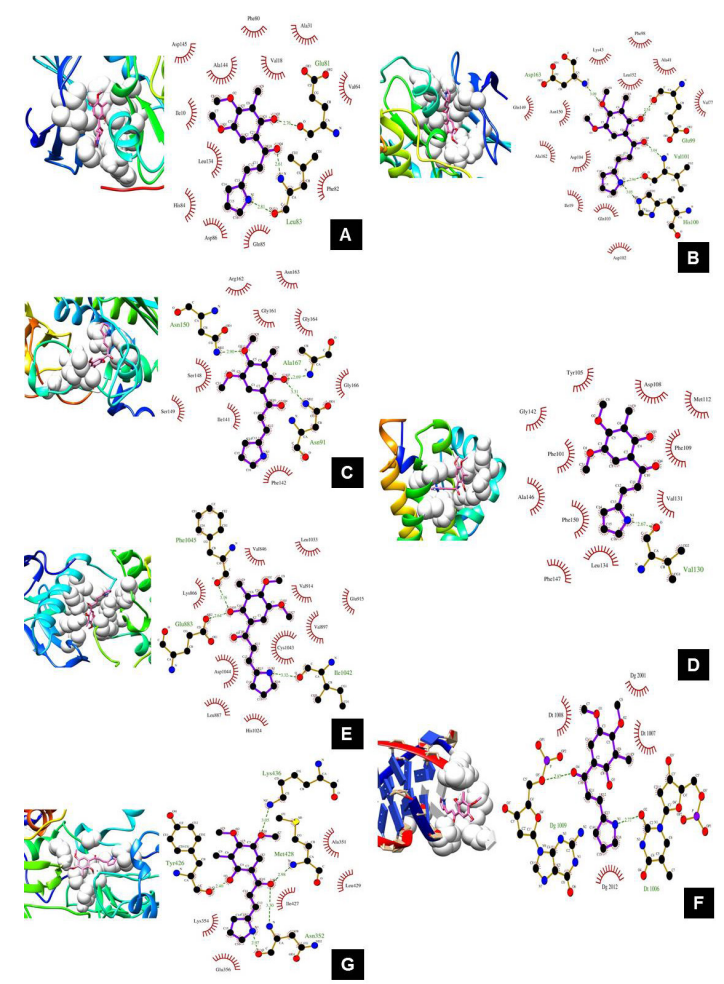

Figure 3. LigPlot + results for molecular interactions between glycopentalone (purple ball and stick) and selected molecular targets CDK-2 (A), CDK-6 (B), Topoisomerase II (C), BCL-2 (D), VEGFR-2 (E), Telomere:Gquadruplex $(\mathbf{F})$, Topoisomerase I (G). Green dashed line indicates hydrogen bond with the labeled distance. Arcs with spikes radiating out corresponds to residues involved in hydrophobic interactions. Binding pockets on the left panel are displayed in white spheres with glycopentalone structure represented in pink stick.

Glycopentalone was docked to CDK-2 and CDK-6 with binding energies of -7.56 and $-7.99 \mathrm{kcal} / \mathrm{mol}$ and inhibition constants of 2.87 and $1.39 \mu \mathrm{M}$, respectively. Both CDK-2 and CDK-6 belong to core-cell cycle machinery and exert their catalytic functions when bound to cyclins. They play crucial roles in cell cycle regulation, apoptosis, transcription, and neuronal functions (Dai and Grant, 2003; Huwe et al., 2003). The favorable binding interaction of glycopentalone with CDK-2 may be attributed to the formation of three hydrogen bonds with bond lengths of 2.76, 2.81, and $2.61 \AA$ via backbone $\mathrm{O}$ atoms of Glu81 and Leu83 and N atom of Leu83, respectively, and hydrophobic interactions were mediated through Ile10, Val18, Ala31, Val64, Phe80, Phe82, His84, Gln85, Leu134, and Ala144. Interestingly, all the hydrogen bonds were found to be established through only backbone $\mathrm{O}$ and $\mathrm{N}$ atoms of the contributing residues. Similarly, glycopentalone showed good interaction with CDK-6. This interaction comprises of five hydrogen bonds with hydrogen-acceptor distances of 2.54, 3.05, 3.04, 2.94, and $3.09 \AA$ through backbone a O atom of Glu99, side chain NE2 atom of His 100, backbone $\mathrm{N}$ and $\mathrm{O}$ atom of Val101, and backbone $\mathrm{N}$ atom of Asp163, respectively, and hydrophobic interactions via Ile19, Ala41, Lys43, Val77, Phe98, Asp102, Gln103, Leu152, and Ala162.

Glycopentalone showed a binding energy of $-6.78 \mathrm{kcal} / \mathrm{mol}$ and an inhibition constant of $10.69 \mu \mathrm{M}$ with Topoisomerase I and with Topoisomerase II it displayed a binding energy of -6.99

Genetics and Molecular Research 15 (3): gmr.15038544 
$\mathrm{kcal} / \mathrm{mol}$ and an inhibition constant of $7.50 \mu \mathrm{M}$. Both DNA Topoisomerases I and II have implicated functions in cell survival and play critical roles in DNA metabolism and structure (Phosrithong and Ungwitayatorn, 2010). Glycopentalone showed fine interaction with Topoisomerase I, which involved five hydrogen bonds of distances 3.30, 2.97, 2.40, 2.98, and 3.05 formed via backbone $\mathrm{N}$ atom and $\mathrm{O}$ atom of Asn352, backbone $\mathrm{O}$ atom of Tyr426, backbone $\mathrm{N}$ atom of Met 428 and side chain NZ atom of Lys436 respectively, and hydrophobic interactions via Ala351, Ala356 and Ile. Glycopentalone also displayed good interaction with Topoisomerase II through the establishment of three hydrogen bonds with hydrogen-acceptor distances via side chain ND2 atom of Asn9, side chain ND2 atom of Asn150 and backbone N atom of Ala167, and hydrophobic interactions via Phe142, Ser148, Ser149, Gly161, Arg162, and Gly164.

Glycopentalone exhibited a binding energy of $-6.38 \mathrm{kcal} / \mathrm{mol}$ and inhibition constant of $20.90 \mu \mathrm{M}$ with Bcl-2. Bcl-2 is an anti-apoptotic oncoprotein, which affects neoplastic cell proliferation by preventing cell death (Reed, 1994). However, glycopentalone did not show a favorable interaction with Bcl-2, which may be understood by the fact that it was able to establish just one hydrogen bond of distance $2.67 \AA$ with backbone O atom of Val130, while a major interaction was contributed by hydrophobic interactions through Phe101, Tyr105, Asp108, Phe109, Leu134, Ala146, Phe147, and Phe150.

Glycopentalone docked to VEGFR-2 with a binding energy of $-8.14 \mathrm{kcal} / \mathrm{mol}$ and inhibition constant of $1.08 \mu \mathrm{M}$. VEGFR-2 is a cell surface receptor for VEGF, expressed highly on vascular endothelial cells and can modulate vascular endothelial survival, proliferation, migration and the formation of vascular tubes (Veikkola et al., 2000). Glycopentalone was able to establish three hydrogen bonds of distances measuring 2.64, 3.32, and $3.16 \AA$ via side chain OE2 atom of Glu883, backbone O atom of Ile1042 and backbone O atom of Phe1045 respectively, and hydrophobic interactions via Val846, Lys866, Leu887, Val897, Val914, His1024, Leu1033, Cys1043, and Asp1044.

Glycopentalone exhibited a decent binding energy of $-8.35 \mathrm{kcal} / \mathrm{mol}$ and inhibition constant of $0.758 \mu \mathrm{M}$ with Telomere G:quadruplex. Telomeres are highly complex nucleoprotein structures at the end of eukaryotic chromosomes, which influence the proliferative capacity of cells. The mammalian telomeric DNA is composed of G-rich tandem repeats (TTAGGG). The bulk of telomeric DNA is double-stranded but the extreme terminus consists of 3 'G-rich single stranded overhang of several hundred bases that act as substrate to which telomeric repeats are added by the enzyme telomerase (Henderson and Blackburn, 1989; Satyanarayana et al., 2004). Glycopentalone was able to establish two hydrogen bonds of distances 2.77 and $2.87 \AA$ via $\mathrm{O} 2$ atoms of a pyrimidine ring of $\mathrm{Dg} 1006$ and O5' atom of phosphate backbone of Dg1009, respectively, and hydrophobic interactions via Dg1007, Dg1008, Dg2001, and Dg2012.

\section{Reverse pharmacophore mapping analysis}

The reverse pharmacophore mapping approach was used to validate the possible targets of glycopentalone suggested by the inverse docking method. Recently, Lei et al. (2015) reported possible targets of 26 isoquinoline alkaloids from Macleaya cordata using the reverse pharmacophore mapping approach, which correlated with their experimentally determined antibacterial, antiparasitic, antitumor, and analgesic effects. We mapped pharmacophore features of glycopentalone against a set of 2241 human target proteins. Of 300 targets identified through Pharmmapper server, we present the top 30 ranked targets for glycopentalone in Table 3.

Genetics and Molecular Research 15 (3): gmr.15038544 


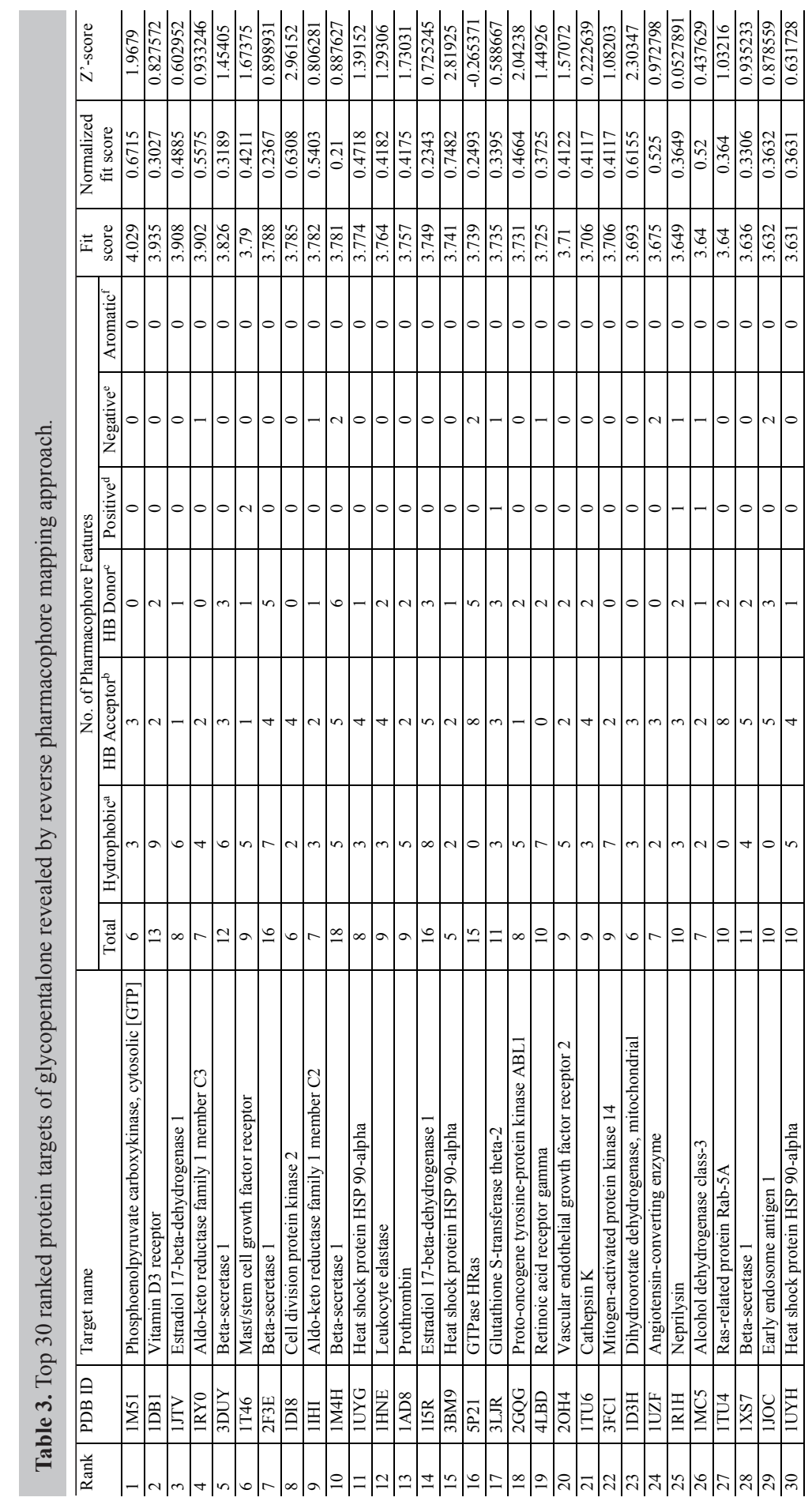

Genetics and Molecular Research 15 (3): gmr.15038544 
Consistent with our molecular docking results, CDK-2 and VEGFR-2 ranked among the top 30 targets. Glycopentalone aligned with the pharmacophore model of CDK2 (Rank 8) with a fit score of 3.785, Z'score of 2.96152 and displayed six pharmacophore features comprising of two hydrophobic centres and four hydrogen bond acceptor (Figure 4A). Similarly, glycopentalone aligned with the pharmacophore model of VEGFR-2 (Rank 20) with a Fit Score of 3.71, Z'score of 1.57072 and displayed nine pharmacophore features comprising of five hydrophobic, two hydrogen bond acceptor and two hydrogen bond donors (Figure 4B). Both the models are statistically significant indicated by large positive Z'-score. Thus, the combined approach of inverse docking and reverse pharmacophore mapping analysis indicates that CDK-2 and VEGFR-2 are the most favorable target for glycopentalone.

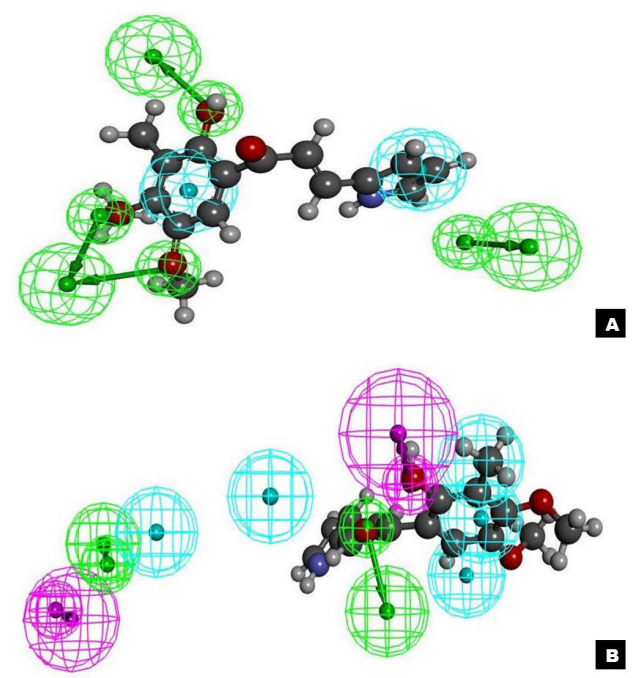

Figure 4. Pharmacophore features of glycopentalone mapped to CDK-2 (A) and VEGFR-2 (B). Structure of glycopentalone is represented in ball and stick model and pharmacophore features are represented in sphereshydrophobic centers (cyan), hydrogen bond acceptors (green), and hydrogen bond donors (pink).

\section{CONCLUSIONS}

The inverse docking analysis of glycopentalone with macromolecules involved in the cell cycle and DNA replication revealed good interactions with Telomere:G-quadruple, VEGFR-2, CDK-6, CDK-2, Topoisomerase II, and Topoisomerase I. We found that glycopentalone's binding energies and inhibition constants were much higher than the bound cocrystal ligands against each molecular target. Glycopentalone showed binding affinities in the descending order as Telomere:G-quadruplex $>$ VEGFR-2 $>$ CDK-6 $>$ CDK-2 $>$ Topoisomerase II $>$ Topoisomerase I $>$ Bcl-2. Glycopentalone displayed binding energies ranging from -6.38 to $-8.35 \mathrm{kcal} / \mathrm{mol}$ and inhibition constants ranging from 0.758 to $20.90 \mu \mathrm{M}$, and exhibited favorable number of hydrogen bonds and hydrophobic interactions with the molecular targets, which indicates its good binding affinity towards the selected molecular targets. The inverse docking suggested targets were validated by a reverse pharmacophore mapping approach, which ranked CDK-2 and VEGFR-2 among the top 30 candidates of 300 possible targets. We found CDK-2 and VEGFR-2 as the most favorable targets of glycopentalone using both

Genetics and Molecular Research 15 (3): gmr.15038544 
approaches, which perhaps gives a reasonable explanation for its experimentally determined cytotoxic and apoptosis promoting effects. Since glycopentalone did not exhibit decent binding energies compared to that of cocrystal ligands, a structure-activity relationship study may be useful in this regard to derive more potent target specific inhibitors. The inverse docking coupled with reverse pharmacophore mapping approach may be of great significance in target fishing of newly discovered small molecules such as natural bioactive compounds.

\section{Conflicts of interest}

The authors declare no conflict of interest.

\section{ACKNOWLEDGMENTS}

The authors would like to extend their sincere appreciation to the Deanship of Scientific Research at King Saud University for its funding of this research through the research group project \#RGP-195.

\section{REFERENCES}

Abbas AM, Abu SM, Shaifuddin A, Sarmina YM, et al. (2011). An evaluation of antimicrobial activities of G. pentaphylla. Res. J. Agric. Biol. Sci. 7: 328-331.

Amran H, Farhana R, Shapna S, Mohammad RR, et al. (2011). Antimicrobial, antioxidant and cytotoxic effects of methanolic extracts of leaves and stems of G. pentaphylla (Retz.) Correa. J. Appl. Pharm. Sci. 8: 137-140.

Chen SJ and Ren JL (2014). Identification of a potential anticancer target of danshensu by inverse docking. Asian Pac. J. Cancer Prev. 15: 111-116. http://dx.doi.org/10.7314/APJCP.2014.15.1.111

Dai Y and Grant S (2003). Cyclin-dependent kinase inhibitors. Curr. Opin. Pharmacol. 3: 362-370. http://dx.doi. org/10.1016/S1471-4892(03)00079-1

DeSantis CE, Lin CC, Mariotto AB, Siegel RL, et al. (2014). Cancer treatment and survivorship statistics, 2014. CA Cancer J. Clin. 64: 252-271. http://dx.doi.org/10.3322/caac.21235

Gan DD, Macaluso M, Cinti C, Khalili K, et al. (2003). How does a normal human cell become a cancer cell? J. Exp. Clin. Cancer Res. 22: 509-516.

Greenlee RT, Murray T, Bolden S and Wingo PA (2000). Cancer statistics, 2000. CA Cancer J. Clin. 50: 7-33. http:// dx.doi.org/10.3322/canjclin.50.1.7

Grinter SZ, Liang Y, Huang SY, Hyder SM, et al. (2011). An inverse docking approach for identifying new potential anticancer targets. J. Mol. Graph. Model. 29: 795-799. http://dx.doi.org/10.1016/j.jmgm.2011.01.002

Gupta N, Agarwal M, Bhatia V, Jha SK, et al. (2011). In vitro antioxidant activity of crude extract of the plant $G$. pentaphylla (Retz.) Correa. Int. J. Pharm. Sci. Rev. Res. 6: 159-162.

Gurung AB, Ali MA, Bhattacharjee A, Abul Farah M, et al. (2016). Molecular docking of anticancer bioactive compound Proceraside with macromolecules involved in cell cycle and DNA replication. Genet. Mol. Res. 15: gmr.15027829.

Halgren TA (1996). Merck molecular force field. I. Basis, form, scope, parameterization, and performance of MMFF94. J. Comput. Chem. 17: 490-519. http://dx.doi.org/10.1002/(SICI)1096-987X(199604)17:5/6<490::AIDJCC1>3.0.CO;2-P

Henderson ER and Blackburn EH (1989). An overhanging 3' terminus is a conserved feature of telomeres. Mol. Cell. Biol. 9: 345-348. http://dx.doi.org/10.1128/MCB.9.1.345

Hughes-Davies L, Caldas C and Wishart GC (2009). Tamoxifen: the drug that came in from the cold. Br. J. Cancer 101: 875-878. http://dx.doi.org/10.1038/sj.bjc.6605231

Huwe A, Mazitschek R and Giannis A (2003). Small molecules as inhibitors of cyclin-dependent kinases. Angew. Chem. Int. Ed. Engl. 42: 2122-2138. http://dx.doi.org/10.1002/anie.200200540

Kharkar PS, Warrier S and Gaud RS (2014). Reverse docking: a powerful tool for drug repositioning and drug rescue. Future Med. Chem. 6: 333-342.http://dx.doi.org/10.4155/fmc.13.207

Lei Q, Liu H, Peng Y and Xiao P (2015). In silico target fishing and pharmacological profiling for the isoquinoline

Genetics and Molecular Research 15 (3): gmr.15038544 
alkaloids of Macleaya cordata (Bo Luo Hui). Chin. Med. 10: 37. http://dx.doi.org/10.1186/s13020-015-0067-4

Liu X, Bai F, Ouyang S, Wang X, et al. (2009). Cyndi: a multi-objective evolution algorithm based method for bioactive molecular conformational generation. BMC Bioinformatics 10: 101. http://dx.doi.org/10.1186/1471-2105-10-101

Liu X, Ouyang S, Yu B, Liu Y, et al. (2010). PharmMapper server: a web server for potential drug target identification using pharmacophore mapping approach. Nucleic Acids Res. 38: W609-14. http://dx.doi.org/10.1093/nar/gkq300

Laskowski RA and Swindells MB (2011). LigPlot+: multiple ligand-protein interaction diagrams for drug discovery. $J$. Chem. Inf. Model. 51: 2778-2786.http://dx.doi.org/10.1021/ci200227u

Mohammed R, Rownak J, Mahbubur R, Syeda S, et al. (2010). A survey of medicinal plants used by folk medicinal practitioners for treatment of gastrointestinal disorders in randomly selected areas of four districts of Bangladesh. Adv. Nat. Appl. Sci. 04: 139-147.

Morris GM, Huey R, Lindstrom W, Sanner MF, et al. (2009). AutoDock4 and AutoDockTools4: Automated docking with selective receptor flexibility. J. Comput. Chem. 30: 2785-2791. http://dx.doi.org/10.1002/jcc.21256

Nayak SS, Jain R and Sahoo AK (2011). Hepatoprotective activity of Glycosmis pentaphylla against paracetamol-induced hepatotoxicity in Swiss albino mice. Pharm. Biol. 49: 111-117.http://dx.doi.org/10.3109/13880209.2010.501084

Pettersen EF, Goddard TD, Huang CC, Couch GS, et al. (2004). UCSF Chimera--a visualization system for exploratory research and analysis. J. Comput. Chem. 25: 1605-1612.http://dx.doi.org/10.1002/jcc.20084

Phosrithong N and Ungwitayatorn J (2010). Molecular docking study on anticancer activity of plant-derived natural products. Med. Chem. Res. 19: 817-835. http://dx.doi.org/10.1007/s00044-009-9233-5

Prawej A, Shofiul A, Reyad-Ul-Ferdous M, Apsana H, et al. (2015). Potential investigation of anti-Inflammatory activity and phytochemical investigations of ethanolic extract of G. pentaphylla leaves. Am. J. Biomed. Res.03: 06-08.

Reed JC (1994). Bcl-2 and the regulation of programmed cell death. J. Cell Biol. 124: 1-6. http://dx.doi.org/10.1083/ jcb.124.1.1

Satyanarayana A, Manns MP and Rudolph KL (2004). Telomeres, telomerase and cancer: an endless search to target the ends. Cell Cycle 3: 1138-1150. http://dx.doi.org/10.4161/cc.3.9.1152

Siegel RL, Miller KD and Jemal A (2015). Cancer statistics, 2015. CA Cancer J. Clin. 65: 5-29. http://dx.doi.org/10.3322/ caac. 21254

Sivakumar M, Chamundeeswari D and Susithra E (2014). Comparative in-vitro antiarthritic studies on the various extracts of G. pentaphylla dc roots. J. Pharm. Res 08: 986-989.

Sreejith PS and Asha VVJ (2015). Glycopentalone, a novel compound from Glycosmis pentaphylla (Retz.) Correa with potent anti-hepatocellular carcinoma activity. J. Ethnopharmacol. 172: 38-43. http://dx.doi.org/10.1016/j. ⿺辶e. 2015.05.051

Sreejith PS, Praseeja RJ and Asha VVJ (2012). A review on the pharmacology and phytochemistry of traditional medicinal plant, G. pentaphylla (Retz.) Correa. J. Pharm. Res 5: 2723.

Stark JL and Powers R (2012). Application of NMR and molecular docking in structure-based drug discovery. Top. Curr. Chem. 326: 1-34. http://dx.doi.org/10.1007/128_2011_213

Veikkola T, Karkkainen M, Claesson-Welsh L and Alitalo K (2000). Regulation of angiogenesis via vascular endothelial growth factor receptors. Cancer Res. 60: 203-212.

Yang H, Tian ST, Wu RY, Chen Y, et al. (2014). Glyco borinine induces apoptosis through mitochondrial pathway in HepG2 cells. J. Asian Nat. Prod. Res. 16: 1-09. http://dx.doi.org/10.1080/10286020.2014.918961

Genetics and Molecular Research 15 (3): gmr.15038544 